Pacific Journal of Mathematic 


\title{
ON GENERALIZED EUCLIDEAN AND \\ NON-EUCLIDEAN SPACES
}

\author{
W. L. Stamey
}

Introduction. The present paper develops necessary and sufficient conditions that a complete, convex, metric space with extendible segments shall be generalized euclidean, $r$-hyperbolic, $r$-spherical, or $r$ elliptic. Blumenthal and others have given four-point conditions which characterize these generalized spaces among certain classes of spaces, and the results of this paper follow the general plan of these earlier works.

1. Definitions, notation and previous results. Unless otherwise noted all terms used have the same meanings as those given in [1]. The distance between two points $p$ and $q$ of a semi-metric space is denoted by $p q$, a point $s$ distinct from $p$ and from $q$ is between $p$ and $q$, denoted by $p s q$, provided $p s+s q=p q$, and a triple of points (not necessarily distinct) is a mid-point triple, denoted by $(p s q)$, provided $p s=s q=$ $p q / 2$. A metric space is said to be generalized \{euclidean, $r$-hyperbolic, $r$-spherical, $r$-elliptic\} provided each of its $n$-dimensional subspaces is congruent with $\left\{E_{n}, H_{n, r}, S_{n, r}, \mathscr{S}_{n, r}\right\}$, where these four symbols represent $n$-dimensional euclidean, hyperbolic, spherical, elliptic space respectively, the last three of space constant $r>0$. A metric space is said to have the weak \{euclidean, $r$-hyperbolic, $r$-spherical, $r$-elliptic\} four-point property provided each of its quadruples containing a triple of points congruent to three points of $\left\{E_{1}, H_{1, r}, S_{1, r}, \mathscr{E}_{1, r}\right\}$ is itself congruent to four points of $\left\{E_{2}, H_{2, r}, S_{2, r}, \mathscr{E}_{2, r}\right\}$. A space has the feeble \{euclidean, $r$ hyperbolic, $r$-spherical, $r$-elliptic $\}$ four-point property provided each quadruple containing a mid-point triple is congruently imbeddable in $\left\{E_{2}, H_{2, r}, S_{2, r}, \mathscr{E}_{2, r}\right\}$. The weak property obviously implies the feeble property.

Theorem 1 (Blumenthal [2]). A complete, convex, externally convex metric space is generalized euclidean if and only if it has the feeble euclidean four-point property.

Defining a conjugate space as one with finite metric diameter $\delta>0$ and having the further property that corresponding to each pair of points $p, q$ of the space with $0<p q<\delta$ there exist points $p^{*}, q^{*}$ of the space with $p q p^{*}, q p q^{*}$, and $p p^{*}=q q^{*}=\delta$ all holding, Hankins [4] has shown the following.

Received June 15, 1956. Presented to the American Mathematical Society April 13, 1956. 
THEOREM 2. If a complete, convex, conjugate, metric space $M$ has diameter $\pi r / 2, r>0$, and if $M$ possesses the feeble $r$-elliptic four-point property, then $M$ is generalized r-elliptic.

2. Metric characterizations. Throughout the remainder of the paper $\Sigma$ will denote a space which satisfies:

(i) $\Sigma$ is metric,

(ii) $\Sigma$ is complete,

(iii) $\Sigma$ is metrically convex,

(iv) if $T_{p, q}$ is a segment with end points $p, q$, there exists $\delta(p)>0$ such that if $s \in T_{p, q}$ with $0<p s<\delta$, then there exists a point $t \in \Sigma$ with (spt) holding.

LEMma 1. If $\Sigma$ has the feeble euclidean, r-hyperbolic, r-elliptic, or $r$-spherical four point property, and if (pqs), $(p q t), q s=q t$, then $s=t$.

Proof. Let $R$ represent any one of the spaces $E_{2}, H_{2, r}, S_{2, r}, \mathscr{E}_{2, r}$. Then $p, q, s, t \approx p_{1}, q_{1}, s_{1}, t_{1} \in R$ and $\left(p_{1} q_{1} s_{1}\right),\left(p_{1} q_{1} t_{1}\right), q_{1} s_{1}=q_{1} t_{1}$ imply that $s_{1}=t_{1}$, so that $s=t$.

REMARK. If in condition (iv) on $\Sigma$ the quantity $\delta(p)$ is unbounded for all $p \in \Sigma$, then $\Sigma$ is externally convex.

THEOREM 1. If $\Sigma$ is externally convex then each two points of $\Sigma$ lie on a unique metric line if and only if pqs, pqt, and $p s=p t$ imply $s=t$.

Proof. The necessity is obvious. The sufficiency is proved by noting that each two points are joined by at least one metric line. Then if there are two distinct segments joining $p$ and $q$, each may be prolonged beyond $q$ along the same segment $T_{q, s}$ to a point $s$, but this implies that $T_{q, s}$ may be prolonged in two distinct ways beyond $q$ to $p$, contrary to hypotheses. Thus $p$ and $q$ must determine a unique segment, and this segment can be prolonged to a metric line in exactly one way.

THEOREM 2. If $\Sigma$ has the feeble euclidean or feeble r-hyperbolic fourpoint property then $\Sigma$ is externally convex.

Proof. Let $p, q \in \Sigma$ with $p \neq q$. Then on a segment $T_{p, q}$ joining $p$ and $q$ choose a point $s$ with $q s>0$ and such that there exists a point $t$ with $(s q t)$. Then denoting either $E_{2}$ or $H_{2, r}$ by $R_{2}$, the hypotheses guarantee that $p, q, s, t \Subset R_{2}$. This together with $p s q$ and $(s q t)$ implies that pqt holds.

THEOREM 3. If $\Sigma$ has the feeble \{euclidean, r-hyperbolic\} four-point 
property, then $\Sigma$ is generalized \{euclidean, r-hyperbolic\}.

Proof. By Theorem $2 \Sigma$ is externally convex and by Lemma 1 (along with the completeness and convexity of $\Sigma$ ) $p q s, p q t, q s=q t$ imply that $s=t$. Thus (Theorem 1) each two points of $\Sigma$ lie on a unique metric line. Then the theorem in the euclidean case is identical with theorem 4.1 in [2]. The $r$-hyperbolic case is handled in the same manner as the euclidean case.

THEOREM 4. If $\Sigma$ has the feeble $r$-spherical four-point property, then $\Sigma$ is a conjugate metric space with metric diameter equal to $\pi r$, and each point $p \in \Sigma$ determines a unique point $p^{*}$ such that $p p^{*}=\pi r$.

Proof. Since $\Sigma$ has the feeble $r$-spherical four-point property, the metric diameter of $\Sigma$ is at most $\pi r$. If $p, q \in \Sigma$ with $0<p q<\pi r$ there exist points $t, v \in \Sigma^{\prime}$ such that $p t q$ and $(t q v)$ hold and $p t+t q+q v<\pi r$. The feeble $r$-spherical four-point property then implies that $p, t, q, v \Subset$ $S_{2, r}$, and this can be strengthened to $p, t, q, v \Subset S_{1, r}$ because $p t q$ and $t q v$ hold.

The feeble $r$-spherical four-point property implies that each pair of points of $\Sigma$ with distance less than $\pi r$ have a unique mid point. This then implies that each two such points are joined by a unique segment. Let $T_{n, q}$ be the segment joining $p$ and $q$, and let $E$ be the set of points $x$ of $\Sigma$ such that $p q x$ holds. All $x \in E$ such that $p x<\pi r$ lie on a unique segment since repeated application of Lemma 1 will show that if $p q x_{1}$, $p q x_{2}, p x_{1}=p x_{2}$, then $x_{1}=x_{2}$. If for $x \in E, \alpha=\operatorname{lub} p x$, then there exists a point $\bar{x} \in E$ such that $p \bar{x}=\alpha$. If $p \bar{x}<\pi r$ there exists a point $y \in E$ such that $p y>\alpha$, so $p \bar{x}=\pi r$.

If there exist two points $p^{*}, p^{* *}$ in $\Sigma$ with $p p^{*}=p p^{* *}=\pi r$, let $q$ be a mid-point of $p^{*}$ and $p^{* *}$. Then $p, p^{*}, p^{* *}, q \approx p_{1}, p_{1}^{*}, p_{1}^{* *}, q_{1} \in S_{2, r}$ and $p_{1} p_{1}^{*}=p_{1} p_{1}^{* *}=\pi r$ gives $p_{1}^{*}=p_{1}^{* *}$ so that $p^{*}=p^{* *}$.

THEOREM 5. If $\Sigma$ has the feeble r-spherical four-point property, then $\Sigma$ has the weak r-spherical four-point property.

Proof. Let $p, q, s, t$ be four points of $\Sigma$ with $p, q, s \Subset S_{1, r}$, to show that $p, q, s, t \Subset S_{2, r}$. If two of the points $p, q, s$ coincide, then $p, q, s, t$ $@ S_{a, r}$, so let it be assumed that $p, q, s$ are pairwise distinct. Then because of the feeble $r$-spherical four-point property some pair, say $p$ and $q$, have distance less than $\pi r$ and determine a unique segment $T_{p, q}$. Let $p, q, s \approx p_{1}, q_{1}, s_{1} \in S_{2, r}$ and let $S_{1, r}\left(p_{1}, q_{1}\right)$ be the unique $S_{1, r}$ determined by $p_{1}$ and $q_{1}$. If $v$ and $v_{1}$ are the unique mid-points of $p, q$ and $p_{1}$, $q_{1}$ respectively, the congruence $p, q, v, t \approx p_{1}, q_{1}, v_{1}, t_{1}$ can be extended 
to $t+T_{p, q} \approx t_{1}+T_{p_{1}, q_{1}}$. If $s \in T_{p, q}, p, q, s, t \in S_{2, r}$. If not, suppose the labelling is such that $q s \leqq p s$, and consider the congruence $q, w, s, q^{*} \approx$ $q_{1}, w_{1}, s_{1}, q_{1}^{*}$, where $w$ is the mid-point of the unique segment $T_{q, q^{*}}$ joining $q, q^{*}$ and containing $s$. This congruence follows from the feeble $r$ spherical four-point property and the free movability of $S_{2, r}$. Then this congruence can be extended to $t+T_{q, q^{*}} \approx t_{1}+T_{q_{1}, q_{1}^{*}}$ and $p, q, s, t \Subset S_{2, r}$.

THEOREM 6. If $\Sigma$ has the feeble r-elliptic four-point property, then $\Sigma$ has metric diameter $\pi r / 2$ and $\Sigma$ is a conjugate space.

Proof. Because of the feeble $r$-elliptic four-point property $\Sigma$ has diameter at most $\pi r / 2$. Let $p, q \in \Sigma$ with $p q<\pi r / 2$. Then there exist points $t, v \in \Sigma$ with $p t q,(t q v)$ holding and $p t+t q+q v<\pi r / 2$. By the feeble $r$-elliptic four-point property $p, t, q, v \Subset \mathscr{E}_{2, r}$ and this can be strengthened to $p, t, q, v \equiv \mathscr{E}_{1, r}$ because of $p t q$ and $(t q v)$.

Let $p, t, q, v \approx p_{1}, t_{1}, q_{1}, v_{1} \in \mathscr{E}_{2, r}$ and let $x \neq v$ and $w$ be points of $\Sigma$ with $p w q$ and $(w q x)$ holding and $p x<\pi r$. Then $p, w, q, x \approx p_{2}, w_{2}, q_{2}$, $x_{2} \in \mathscr{E}_{2, r}$ and $p_{2}, w_{2}, q_{2}, x_{2}$ lie on an $\mathscr{E}_{1, r}$. Then there exists a motion of $\mathscr{E}_{2, r}$ sending $p_{2}, q_{2}$ into $p_{1}, q_{1}$ respectively and sending $w_{2}$ and $x_{2}$ into uniquely determined points $w_{1}$ and $x_{1}$ on the $\mathscr{E}_{1, r}$ determined by $p_{1}$ and $q_{1}$. Thus if $M$ is the set of $x \in \Sigma$ with $p q x$ and $p x<\pi r$ holding, the unique segment $T_{p, q}$ can be uniquely extended to $T_{p, q} \cup T_{q, x}=T_{p, x}$ for $x \in M$.

Let now $\alpha=\operatorname{lub} p x$ for $x \in M$ and let $\left\{x_{i}\right\}$ be a sequence of points of $M$ such that $\lim p x=\alpha$ and if $i<j, p x_{i} x_{;}$holds. Then since $p$ and all of the $x_{i}$ lie on the same metric segment, as $i, j \rightarrow \infty, x_{i} x_{j} \rightarrow 0$. The completeness of $\Sigma$ then implies the existence of a point $y$ such that $y=\lim x_{i}$ and $p y=\alpha \leq \pi r / 2$. Furthermore, since $p q x_{i}$ holds for $i=1,2$, ... and $\Sigma$ is metric, $p q y$ holds. If $p y<\pi r / 2$, then $y \in M$ and there exists $y \in \Sigma$ such that $p \bar{y}>p y=\alpha$, and this is impossible.

Finally the uniqueness of extensions of segments insures that if $p p^{*}=p p^{* *}=\pi r / 2$ and $p q p^{*}, p q p^{* * *}$ hold, then $p^{*}=p^{* *}$.

THEOREM 7. If $\Sigma$ has the feeble $\{r$-spherical, r-elliptic $\}$ four-point property, then $\Sigma$ is generalized $\{r$-spherical, r-elliptic $\}$.

Proof. The theorem follows in the spherical case from Theorem 5 upon application of Theorem 66.5 of [1] and in the elliptic case from Theorem 6 and Theorem 4.4 of [3].

M. M. Day ${ }^{1}$ [3] has defined another four-point property which he calls the "queasy euclidean four-point property" and has shown that a

1 The author is indebted to the referee for calling his attention to Day's work and for suggesting the possibility of the extension of Day's work. 
complete, externally convex semimetric space possessing this property is generalized euclidean. The remainder of this paper is devoted to extending Day's work.

A semimetric space $M$ will be said to have the queasy \{euclidean, $r$-hyperbolic, $r$-spherical $\}$ four-point property provided that corresponding to each pair of points $p, s \in M$ there exists $q \in M$ such that $p q s$ holds and for each $t \in M$, the quadruple $p, q, s, t \in\left\{E_{2}, H_{2, r}, S_{2, r}\right\}$.

Lemma 2. If $\Sigma$ has the \{euclidean, r-hyperbolic\} four-point property, then each two distinct points of $\Sigma$ are joined by a unique metric segment.

Proof. Since $\Sigma$ is complete, convex and metric each two points are joined by at least one segment. It will be sufficient then to show that each pair of points of $\Sigma$ have just one mid-point. Let $p, q_{1}, q_{2}, s \in \Sigma$ with $\left(p q_{1} s\right),\left(p q_{2} s\right)$ and $p \neq s$ holding, and let $R$ represent either of the spaces $E_{2}$ or $H_{2, r}$. If there exists a sequence of points $t_{i} \in \Sigma, i=1,2$, $\cdots$, with $\lim t_{i}=q_{1}, p t_{i} q_{1}, p t_{i} q_{2}$ holding, then $\lim t_{i}=q_{2}$ and $q_{1}=q_{2}$.

If $q_{1} \neq q_{2}$, then there exists a positive number $\bar{\rho}_{1}$ such that if $p t+$ $t q_{1}=p q_{1}$ and $p t+t q_{2}=p q_{2}$ then $t q_{1}=t q_{2}>\bar{\rho}_{1}$. Also there exists $\bar{\rho}_{2}>0$ such that if $q_{1} t+t s=q_{1} s$ and $q_{2} t+t s=q_{2} s$, then $t q_{1}=t q_{2}>\bar{\rho}_{2}$. Let $\rho_{1}$ be the least upper bound of the numbers $\bar{\rho}_{1}$ and $\rho_{2}$ that of the numbers $\bar{\rho}_{z}$. Let $\bar{p}$ and $p^{*}$ be points of $\Sigma$ with $p \bar{p}+\bar{p} q_{1}=p q_{1}, p p^{*}+p^{*} q_{2}=p q_{2}$ and $\bar{p} q_{1}=p^{*} q_{2}=$ $\rho_{1}$. Then either $\bar{p}=p^{*}$ or there is a sequence $p_{i}$ with $p p_{i} q_{1}, p p_{i} q_{2}$ holding and $\lim p_{i}=\bar{p}, \lim p_{i}=p^{*}$ so that $\bar{p}=p^{*}$. Thus there exist two points of $\Sigma$ with $q_{1}$ and $q_{2}$ each metrically between these points but such that any segment joining the points and containing $q_{1}$ has only end points in common with a segment joining these points and containing $q_{2}$. There will be no loss of generality if these points are taken to be $p$ and $s$ and if $q_{1}$ and $q_{2}$ are assumed to be distinct middle points of $p$ and $s$.

The queasy four-point property of $\Sigma$ implies that there exist $x \in \Sigma$, $\bar{p}, \bar{x}, \bar{q}, \bar{s}, p^{*}, x^{*}, q^{*}, s^{*} \in R$ with $p x s$ holding and

$$
\begin{aligned}
& p, x, q_{1}, s \approx \bar{p}, \bar{x}, \bar{q}, \bar{s} \\
& p, x, q_{2}, s \approx p^{*}, x^{*}, q^{*}, s^{*} .
\end{aligned}
$$

Then since $p^{*} x^{*} s^{*}$ and $p^{*} q^{*} s^{*}$ hold, there is a motion sending the "starred" points into the corresponding "barred" ones, and $\bar{p}, \bar{x}, \bar{q}, \bar{s}$ all lie on one metric segment of $R$. Thus either $\bar{x}=\bar{q}$ and $x=q_{1}=q_{2}$ or there is a metric segment joining $p, q_{1}, s$ and one joining $p, q_{2}, s$ with these two segments having interior point $x$ in common. This contradiction completes the proof.

LEMMA 3. If $\Sigma$ has the queasy r-spherical four-point property, then each two distinct points having distance less than $\pi r$ are joined by a unique segment. 
Proof. The proof is identical with that of the preceeding lemma if distance $p s$ is restricted to be less than $\pi r$.

THEOREM 8. If $\Sigma$ has the queasy \{euclidean, r-hyperbolic, r-spherical\} four point property, then $\Sigma$ is generalized \{euclidean, r-hyperbolic, $r$-spherical\}.

Proof. It will be sufficient to show that if $p, q, s, t \in \Sigma$ with (pqs) holding, then $p, q, s, t \in R$, where $R$ represents any one of the spaces $E_{2}, H_{2, r}, S_{2, r}$. Assume for the present that if $R$ is spherical, $p s<\pi r$. Then let $x \in R$ with $p x s$ holding and

$$
\begin{aligned}
& p, x, q, s \approx \bar{p}, \bar{x}, \bar{q}, \bar{s} \in R \\
& p, x, s, t \approx p^{*}, x^{*}, s^{*}, t^{*} \in R
\end{aligned}
$$

Then there exists a motion of $R$ sending $p^{*}, s^{*}$ into $\bar{p}, \widetilde{s}$ respectively and $t^{*}$ into a point $\bar{t}$. If $q t=\overline{q t}$, then $p, q, s, t \approx \bar{p}, \bar{q}, \bar{s}, \bar{t}$.

If $q t \neq \overline{q t}$, let a congruence $f$ between the segments $T_{p, s}$ and $T_{\vec{p}, \dot{s}}$ be established so that $f(p)=\bar{p}, f(s)=\bar{s}$. Let $Q$ represent the set of points $x \in T_{p, s}$ such that $t x=\bar{t} f(x)$. Then the continuity of the metric in $\Sigma$ implies that in traversing $T_{p, s}$ from $p$ to $q$ there is a last point of $Q$ encountered. Let this point be $u$, and let $w$ be the last point of $Q$ encountered in traversing $T_{p, s}$ from $s$ toward $q$. Denote $\bar{u}=f(u), \bar{w}=$ $f(w)$.

Then there exists by the queasy property a point $y \in \Sigma$ with $u y w$ holding and $u, y, w, t \approx u^{\prime}, y^{\prime}, w^{\prime}, t^{\prime} \in R$. A motion of $R$ sends $u^{\prime}, w^{\prime}, t^{\prime}$ into $\bar{u}, \bar{w}, \bar{t}$ and $y^{\prime}$ into a unique point $\bar{y}$ with $\overline{u y w}$ holding and $\overline{y t}=y^{\prime} t^{\prime}$ $=y t$. This contradicts the property used to pick out $u$ and $w$ so that $q t=\overline{q t}$ and $p, q, s, t \approx R$.

Finally if $R$ represents $S_{2, r}$ and $p s=\pi r$, there is a point $x \in \Sigma$ with pxs holding and

$$
\begin{aligned}
& p, x, s, q \approx \bar{p}, \bar{x}, \bar{s}, \bar{q} \in S_{2, r} \\
& p, x, s, t \approx p^{*}, x^{*}, s^{*}, t^{*} \in S_{2, r} .
\end{aligned}
$$

Let a motion be performed sending $p^{*}, s^{*}$ into $\bar{p}, \bar{s}$ respectively and $t^{*}$ into a point $\bar{t}$. Consider the set of distances $\bar{t} x$ where $x$ belongs to the $S_{1, r}$ at distance $\pi r / 2$ from $p$. This set of numbers has a minimum $m$ and a maximum $M$. Let the labelling be taken so that $p t \leqq \pi r / 2$. Then it is necessary that $m \leqq t q \leqq M$. For if $t q<m$, then $p t+m=\pi r / 2$ and $p t+t q<\pi r / 2=p q$. Also if $t q>M, t q>t p+p q$.

Now on the $S_{1, r}$ at distance $\pi r / 2$ from $p$ there is a point $\overline{\bar{q}}$ so that $\bar{t} \overline{\bar{q}}=t q$. Then $p, q, s, t \approx \bar{p}, \overline{\bar{q}}, \bar{s}, \bar{t}$, and this completes the proof. 
Of course the proof of Theorem 8 is not valid for $\mathscr{E}_{2, r}$ because of the strong use made of free movability. It should also be noted that when the queasy four-point property is assumed for a semi-metric space, it is unnecessary to assume convexity and metricity since the queasy property implies these.

\section{REFERENCES}

1. L. M. Blumenthal, Theory and applications of distance geometry, Oxford, Clarendon Press, 1953.

2. , An extension of a theorem of Jurdan and von Neumann, Pacific J. Math., 5, (1955), 161-167.

3. M. M. Day, Some Criteria for inner product spaces. (Abstract), Bull. Amer. Math. Soc., 62, (1956), 45-46.

4. J. D. Hankins, Metric characterizations of elliptic $n$-space, University of Missouri doctoral dissertation (1954).

Kansas State College 



\title{
PACIFIC JOURNAL OF MATHEMATICS
}

\author{
EDITORS
}

H. L. Royden

Stanford University

Stanford, California

R. A. Beaumont

University of Washington

Seattle 5, Washington
A. L. Whiteman

University of Southern California

Los Angeles 7, California

E. G. Straus

Unıversity of California

Los Angeles 24, California

\section{ASSOCIATE EDITORS}

\author{
E. F. BECKENBACH \\ C. E. BURGESS \\ M. HALL \\ E. HEWITT
}

\author{
A. HORN \\ V. GANAPATHY IYER \\ R. D. JAMES \\ M. S. KNEBELMAN
}

L. NACHBIN
I. NIVEN
T. G. OSTROM
M. M. SCHIFFER

G. SZEKERES

F. WOLF

K. YOSIDA

\section{SUPPORTING INSTITUTIONS}

UNIVERSITY OF BRITISH COLUMBIA

CALIFORNIA INSTITUTE OF TECHNOLOGY

UNIVERSITY OF CALIFORNIA

MON'TANA STATE UNIVERSITY

UNIVERSITY OF NEVADA

OREGON STATE COLLEGE

UNIVERSITY OF OREGON

UNIVERSITY OF SOUTHERN CALIFORNIA

\author{
STANFORD UNIVERSITY \\ UNIVERSITY OF UTAH \\ WASHINGTON STATE COLLEGE \\ UNIVERSITY OF WASHINGTON \\ AMERICAN MATHEMATICAL SOCIETY \\ CALIFORNIA RESEARCH CORPORATION \\ HUGHES AIRCRAFT COMPANY \\ THE RAMO-WOOLDRIDGE CORPORATION
}

Mathematical papers intended for publication in the Pacific Journal of Mathematics should be typewritten (double spaced), and the author should keep a complete copy. Manuscripts may be sent to any of the editors. All other communications to the editors should be addressed to the managing editor, E. G. Straus at the University of California, Los Angeles 24, California.

50 reprints per author of each article are furnished free of charge; additional copies may be obtained at cost in multiples of 50 .

The Pacific Journal of Mathematics is published quarterly, in March, June, September, and December. The price per volume (4 numbers) is $\$ 12.00$; single issues, $\$ 3.50$. Back numbers are available. Special price to individual faculty members of supporting institutions and to individual members of the American Mathematical Society: $\$ 4.00$ per volume; single issues, $\$ 1.25$.

Subscriptions, orders for back numbers, and changes of address should be sent to Pacific Journal of Mathematics, 2120 Oxford Street, Berkeley 4, California.

Printed at Kokusai Bunken Insatsusha (International Academic Printing Co., Ltd.), No. 10, 1-chome, Fujimi-cho, Chiyoda-ku, Tokyo, Japan.

PUBLISHED BY PACIFIC JOURNAL OF MATHEMATICS, A NON-PROFIT CORPORATION

The Supporting Institutions listed above contribute to the cost of publication of this Journal, but they are not owners or publishers and have no responsibility for its content or policies. 


\section{Pacific Journal of Mathematics}

\section{Vol. 7, No. $3 \quad$ March, 1957}

Silvio Aurora, Multiplicative norms for metric rings............... 1279

Ross A. Beaumont and John Richard Byrne, On the construction of

$R$-modules and rings with polynomial multiplication ............ 1305

Fred Brafman, An ultraspherical generating function . . . . . . . . . . . 1319

Howard Ernest Campbell, On the Casimir operator ............... 1325

Robert E. Edwards, Representation theorems for certain functional

operators..................................... 1333

Tomlinson Fort, The five-point difference equation with periodic

coefficients ..................................... 1341

Isidore Heller, On linear systems with integral valued solutions......... 1351

Harry Hochstadt, Addition theorems for solutions of the wave equation in

parabolic coordinates ................................ 1365

James A. Hummel, The coefficient regions of starlike functions . . . . . . . . 1381

Fulton Koehler, Estimates for the eigenvalues of infinite matrices ......... 1391

Henry Paul Kramer, Perturbation of differential operators ............. 1405

R. Sherman Lehman, Development of the mapping function at an analytic

corner .......................................... 1437

Harold Willis Milnes, Convexity of Orlicz spaces.................. 1451

Vikramaditya Singh, Interior variations and some extremal problems for certain classes of univalent functions . . . . . . . . . . . . . . . . 1485

William Lee Stamey, On generalized euclidean and non-euclidean spaces ............................................. 1505

Alexander Doniphan Wallace, Retractions in semigroups .............. 1513

R. L. Wilder, Monotone mappings of manifolds .................. 1519 